\title{
Cystic Fibrosis Transmembrane Conductance Regulator (CFTR) Gene Mutations and Risk for Pancreatic Adenocarcinoma
}

\author{
Robert R. McWilliams, M.D., Gloria M. Petersen, Ph.D., Kari G. Rabe, M.S., Leonard M. \\ Holtegaard, Pamela J. Lynch, Michele D. Bishop, M.D., and W. Edward Highsmith Jr, Ph.D. \\ From the Departments of Oncology (R.M), the Division of Gastroenterology and Hepatology (G.P.), \\ the Division of Epidemiology (G.P.), the Division of Laboratory Genetics (L.H., P.L., W.H.), and the \\ Division of Biostatistics (K.R.), Mayo Clinic, Rochester, Minnesota, and the Division of \\ Gastroenterology and Hepatology (M.B.), Mayo Clinic, Jacksonville, Florida.
}

\begin{abstract}
Background-Mutations in the cystic fibrosis transmembrane conductance regulator (CFTR) gene are common in white persons and are associated with pancreatic disease. The purpose of this casecontrol study was to determine whether CFTR mutations confer a higher risk of pancreatic cancer.
\end{abstract}

Methods-In a case-control study, we compared the rates of 39 common cystic fibrosis-associated CFTR mutations between 949 white patients with pancreatic adenocarcinoma and 13,340 white controls from a clinical laboratory database for prenatal testing for CFTR mutations. The main outcome measure was the CFTR mutation frequency in patients and controls.

Results-Overall, 50 (5.3\%) of 949 patients with pancreatic cancer carried a common CFTR mutation versus 510 (3.8\%) of 13,340 controls (odds ratio, 1.40; 95\% confidence interval, 1.04-1.89; $P=.027)$. Among patients who were younger when their disease was diagnosed ( $<60$ years), the carrier frequency was higher than in controls (odds ratio, $1.82 ; 95 \% \mathrm{CI}, 1.14-2.94 ; P=.011$ ). In patient-only analyses, the presence of a mutation was associated with younger age (median 62 vs 67 years; $P=$. 034). In subgroups, the difference was seen only among ever-smokers, (60 vs 65 years, $\mathrm{p}=.028$ ). Subsequent sequencing analysis of the CFTR gene detected 8 (16\%) compound heterozygotes among the 50 patients initially detected to have 1 mutation.

Conclusions-Carrying a disease-associated mutation in CFTR is associated with a modest increase in risk for pancreatic cancer. Those affected appear to be diagnosed at a younger age, especially among smokers. Clinical evidence of antecedent pancreatitis was uncommon among both carriers and noncarriers of CFTR mutations.

\section{Keywords}

Pancreatic neoplasms; molecular epidemiology; cystic fibrosis transmembrane conductance regulator

\section{INTRODUCTION}

Pancreatic cancer is a common, highly lethal malignancy $(1,2)$. Of the approximately 37,000 cases diagnosed annually in the United States, $5 \%$ to $10 \%$ report a family history of pancreatic cancer, and high penetrance genetic factors are being explored (3). Because the majority of

Address reprint requests to Robert R. McWilliams, MD, Department of Oncology, Mayo Clinic, 200 First Street SW, Rochester, MN 55905. E-mail: mcwilliams.robert@mayo.edu.

The authors have no conflicts of interest to disclose. 
those affected have no family history, a risk profile of low-penetrance genes for "sporadic" pancreatic adenocarcinoma would be valuable when incorporated into development of strategies for prevention or screening.

Mutations in the cystic fibrosis transmembrane regulator (CFTR) gene have been found to be associated with chronic pancreatitis, with the mechanism likely being secondary to ion regulator dysfunction in pancreatic ductal cells impairing drainage (4). Notably, pancreatitis is diagnosed at a young age (mean, 22.7 years; range, 10-35 years) among CFTR mutation carriers (5). It has been reported that patients with chronic pancreatitis have up to a 26 -fold higher risk for pancreatic cancer than does the general population (6). Several patients with known cystic fibrosis (CF) have developed pancreatic adenocarcinoma at ages ranging from 23 to 39 years (7-9), much younger than ages typically reported for pancreatic cancer (10). Two cohort studies have shown an increased risk for pancreatic cancer among patients with CF $(11,12)$. Therefore, we hypothesized that the increased risk of pancreatitis due to CFTR mutations may confer a higher risk of adenocarcinoma of the pancreas in carriers than in noncarriers.

We previously reported a pilot study showing a higher than expected frequency of CFTR mutations in 166 pancreatic cancer patients diagnosed younger than 60 years of age (13). To explore further the relationship of CFTR gene mutations and pancreatic cancer, we extended the analysis to include concurrent patients of older ages and augmented the size of our sample with additional patients since the original report. This report now includes all consecutively recruited cases among whites over a 5+ year time period. With this study, we aimed to determine whether carrying a muattions in $C F T R$ was associated with an overall increased risk for pancreatic cancer.

\section{METHODS}

\section{Patients}

From October 2000 to February 2006, a total of 1,060 patients with histologically proven (96\%) or clinically diagnosed pancreatic adenocarcinoma of all stages consented to participate in a prospective registry at the time of their Mayo Clinic visit (ultrarapid recruitment), as previously described (14). This approach resulted in a 66\% participation rate among all identified patients seen at Mayo Clinic with pancreatic adenocarcinoma of any stage. Written, informed consent was obtained from each subject for participation in the registry study and provision of biospecimens, and this study was approved by the Mayo Clinic Institutional Review Board, including the use of case samples from the registry and control sample data from Mayo Medical Laboratories. On enrollment, patients were asked to complete a risk-factor questionnaire. DNA was collected from peripheral blood for analysis on 970 potentially eligible patients. When questionnaire data were unavailable $(26.2 \%)$, medical record data were used to obtain pancreatitis, family history, and smoking information. Clinical diagnosis required a malignantappearing pancreatic mass and at least 2 of the following characteristics: elevated carbohydrate antigen 19-9, jaundice, weight loss, or abdominal pain. Because nearly all cases were in whites $(97.8 \%)$, in order to avoid population stratification, nonwhite subjects were excluded from both case and control groups. Both Hispanic whites $(0.9 \%$ of those with ethnicity information available) and non-Hispanic whites $(92.5 \%)$ were included, with ethnicity unavailable on 62 $(6.5 \%)$. A total of 949 patients were included in this study.

\section{Controls}

Mayo Medical Laboratories provides clinical laboratory testing for CFTR mutations; reasons for testing include suspected diagnosis of $\mathrm{CF}$, carrier status determination to inform genetic 
counseling, and prenatal or prepregnancy parental screening. Specimens are received from medical facilities nationwide.

For our control group, we used the results from 1 year's testing (April 2006-March 2007) by Mayo Medical Laboratories. Included were persons older than 18 years who self-reported white, northern European, or mixed European race. We excluded samples drawn for suspected CF or family history of CF. In total, 13,340 controls were included.

\section{Genotyping}

All control samples and 797 of 949 patient samples were analyzed for CFTR mutations using a universal array-based, commercially available test kit (Tag-It Mutation Detection Kit for CFTR 40+4; Luminex Corporation, Austin, Texas) (15). This assay screens for 23 mutations recommended for population screening by the American College of Medical Genetics (16), plus 16 additional mutations found in CF patients. For the remaining 152 patient samples, we used a commercially available, analyte-specific reagent for prenatal screening, counseling, and diagnosis (Cystic Fibrosis v3.0 ASR; Celera, Alameda, California). No mutations were detected in these samples that were not analyzed by the universal array method, and prior studies including more than 1,000 patients have demonstrated $100 \%$ concordance between the 2 methods $(17,18)$.

\section{Sequencing}

All positive samples $(\mathrm{n}=50)$ were analyzed for a second CFTR mutation. All 27 exons of the gene were amplified in 8 multiplex polymerase chain reaction analyses, and each exon was sequenced in both forward and reverse directions using internal sequencing primers and a commercially available sequencing kit (BigDye Terminator v1.1 Cycle Sequencing Kit; Applied Biosystems, Valencia, California). Capillary electrophoresis was carried out on an ABI 3730 instrument (Applied Biosystems, Inc, Foster City, California), and the data were analyzed using Mutation Surveyor, Version 2.41 (Softgenetics, College Station, Pennsylvania

\section{Clinical Variables}

Questionnaires completed by pancreatic cancer patients were used for secondary case-only analyses comparing mutation carrier and noncarrier cases by smoking status, family history of pancreatic cancer in first-degree relatives, and antecedent pancreatitis. Individuals who reported smoking 100 or more cigarettes in their lifetime were classified as "ever-smokers," and those who reported fewer than 100 cigarettes in their lifetime were classified as "neversmokers." Given the similarity of symptoms between pancreatitis and pancreatic cancer, only pancreatitis diagnoses made more than 3 years before cancer diagnoses were included in the analyses. Of the 949 cases, 705 completed the risk factor questionnaires. Completion of missing variables on the remaining 244 that were extractable from the medical record was accomplished by a specialist physician (R.R.M.). To validate this source of data, records on 25 randomly selected patients who had completed questionnaires were abstracted as well, with the abstractor blinded to the questionnaire information. Kappa statistics were generated for the following variables: ever/never smoker (0.92), presence of pancreatitis ( 0.43$)$, first-degree family history of pancreatic cancer (0.65), race (1.0), and ethnicity (1.0).

\section{Statistical Methods}

Frequencies of mutations were determined in both the patients with pancreatic cancer and the controls and compared using $\chi^{2}$ statistical tests. Because of the large differences in age and sex between the patient group and the control group, multivariate analyses for age and sex were not performed, as these differences would falsely skew the comparison. Within the patient group, comparisons of age of diagnosis, smoking status, frequency of pancreatitis, and family 
history of pancreatic cancer were compared among carriers using $\chi^{2}$ or Kaplan-Meier plots and log-rank tests. As this study was testing a specific hypothesis, there was no correction undertaken for multiple comparisons.

\section{RESULTS}

The median age of pancreatic cancer patients was 67 years (range, 28-91 years), and 58\% were male. Twenty-eight percent of patients had pancreatic tumors that were surgically resectable, and $34 \%$ of tumors were locally advanced; $38 \%$ of patients had distant metastases at enrollment. Controls were a median age of 27 years (range, 18-81 years), and 4\% were male. CFTR mutation frequencies in white pancreatic cancer patients and controls are compared in Table 1.

Overall, $50(5.3 \%)$ of 949 patients had a CFTR mutation detected by the multisite assays versus $510(3.8 \%)$ of 13,340 controls (odds ratio [OR], 1.40; 95\% confidence interval [CI], 1.04-1.89; $P=.027)$. Seven different $C F T R$ mutations were detected among 949 cases, and 24 different

CFTR mutations were detected among 13,340 controls. Interestingly, $10 \%$ of carriers among cases carried W1282X compared with $1 \%$ of controls. This is a mutation overrepresented in the Ashkenazi Jewish population (20). However, of the 5 cases with W1282X mutations detected, only 2 reported Jewish heritage, and neither responded whether they were Ashkenazi. The 3 remaining carriers reported their religion as Christian. None of 7 self-reported Ashkenazi Jewish cases had mutations detected. No compound heterozygotes or homozygotes for mutations were found among patients or controls by the initial genotyping assays.

When carrier rates of detected mutations among patients with pancreatic cancer diagnosed at age younger than 60 years $(\mathrm{n}=281)$ were compared with carrier rates for controls, the OR increased to 1.83 (95\% CI, 1.14-2.94; $P=.011)$. For cases younger than 55 years $(\mathrm{n}=159)$, the OR was 1.34 (95\% CI, 0.65-2.74), and for cases younger than 50 years $(n=74)$, the OR was 1.83 (95\% CI, 0.73-4.55). A detected mutation was associated with younger median age at cancer diagnosis (62 years) compared with a median age of 67 years in those without detected mutations $(P=.034)$. Notably, younger age at diagnosis was most marked in mutation carriers who ever smoked (Figure 1). The median age of diagnosis in 29 mutation carriers who ever smoked was 60 years versus 65 years for 545 ever-smoker noncarriers $(P=.028)$, while for never-smokers showed no difference in age of diagnosis (21 carriers vs 351 noncarriers), where the median age in carriers was 69 years versus 68 years for noncarriers $(P=.32)$. Finer breakdowns of smoking such as smoking intensity and current smoking were not performed due to extremely low numbers of mutation carriers in these finer analyses.

Self-reported pancreatitis predating the cancer diagnosis by at least 3 years was rare overall and not significantly higher $(P=.75)$ among mutation carriers $(2.0 \%)$ than noncarriers $(1.5 \%)$. Pancreatitis in 14 patients did not appear to affect age of diagnosis of cancer; the median age of diagnosis was 68.5 years in those affected compared with 67 years in those without pancreatitis $(P=.55)$. Carrier cases were more likely to have reported a first-degree family history of pancreatic cancer than noncarrier cases $(8.0 \%$ vs $6.5 \%)$, but this difference was not statistically significant $(P=.67)$.

All detected carriers in the pancreatic cancer group underwent whole gene sequencing to determine the frequency of compound heterozygosity. Additional variants were detected in 8 $(16 \%)$ of the 50 carriers (Table 2). Age at diagnosis was older in these compound heterozygotes compared with those carrying only 1 mutation (71.5 vs 62 years), although this difference was not statistically significant $(P=.22)$. 


\section{DISCUSSION}

The relationship of CFTR gene mutations and pancreatic cancer has been explored in 2 prior studies, and the reported associations were null. However, we believe these studies were not comprehensive enough or adequately powered to detect a modest association. Malats et al (21) assessed the most common CFTR mutation site ( $\triangle \mathrm{F} 508)$ in a Spanish population of 128 patients with exocrine pancreatic cancer. Carrier frequencies of the $\Delta \mathrm{F} 508$ mutation and $5 \mathrm{~T}$ polymorphisms in cancer patients ( $2.3 \%$ and $5.5 \%$, respectively) were not higher than expected for the Spanish population.

In 2003, Matsubayashi et al (22) analyzed 334 surgically treated sporadic pancreatic cancer patients for mutations in $\Delta \mathrm{F} 508$ and $5 \mathrm{~T}$ polymorphism. Among these, $2.5 \%$ had the $\Delta \mathrm{F} 508$ mutation and $9.3 \%$ had the $5 \mathrm{~T}$ polymorphism, again similar to background population rates.

These reports provide only snapshots in assessing a major effect on risk and did not fully address the question of $C F T R$ involvement in risk for pancreatic cancer. Only 2 sites in the CFTR gene (1 mutation, 1 polymorphism) were examined in the 2 studies, but more than 1,000 different mutations have been reported to be involved in CF (23). No normal control group was tested in either analysis.

One potential mechanism for cancer development in CFTR mutation carriers is chronic pancreatitis, a known risk factor for pancreatic cancer. However, the finding that so few ( 1 of 50) of our CFTR carriers had an antecedent history of pancreatitis is intriguing. Either the pancreatitis is subclinical, as has been described in alcoholic patients (24), or the presence of a single mutant CFTR allele may increase the risk for pancreatic cancer through a mechanism independent of chronic pancreatitis or inflammation. Histologic or radiographic identification of patients with pancreatitis was not performed, since underlying antecedent pancreatitis is indistinguishable from postobstructive pancreatitis as a result of the primary tumor.

In mutation carriers, the relationship between younger age of cancer diagnosis and smoking is notable: smokers were younger at diagnosis if they carried a mutation, whereas there was no difference in nonsmokers. In patients with hereditary pancreatitis, smoking increases risk for pancreatic cancer and lowers the age of diagnosis by 20 years (25). The additive effect on age at diagnosis of tobacco exposure across different genetic mechanisms adds validity to our association of CFTR and younger age at diagnosis. This finding underscores the need to counsel patients with pancreatitis or known genetic risk factors for pancreatitis to avoid tobacco use.

The importance of other coding variants detected by our sequencing analysis is unclear, since none of the patients with "genetic" CF carried that diagnosis clinically. S42F has been described in 1 Italian CF patient (26). E528E is a synonymous polymorphism involving the last base pair in exon $10(1716 \mathrm{G}>\mathrm{A})$, which has been reported to affect splicing, but has not been linked to severe pulmonary disease (27). S912L is thought to be a neutral variant (serine for a leucine), unless in cis position to another mutation (28). The F1052V missense mutation is a variant with a modest effect, with normal or near-normal sweat chloride tests in combination with other mild variants $(29,30)$. N1088S is a novel mutation that substitutes asparagine for a serine amino acid, both positively charged, and its functionality is unclear. The mutation M1191I is also novel, with a substitution of a hydrophobic amino acid for another (threonine for isoleucine). S1235R has been variably reported in CF patients, but phenotype is more severe in association with second cis mutations $(31,32)$. The $5 \mathrm{~T}$ poly-T polymorphism affects splicing of exon 9 (33) and contributes to congenital bilateral absence of the vas deferens (34) and possibly pancreatitis (4), but not pulmonary disease. It is difficult to determine whether there is an excess of compound heterozygotes detected (16\%). In 24 idiopathic pancreatitis patients reported by Bishop et al (35) to carry CFTR mutations, $25 \%$ were compound heterozygotes. However, among 50 healthy blood donors, $22 \%$ had 1 variant detected by sequencing. 
Similarly, in a slightly larger sequencing study, Weiss et al.found CFTR mutations in 11 of 60 controls (18.3\%).(36) Neither study reported compound heterozygotes in "healthy" controls, though the numbers were insufficient to draw substantial conclusions. Taking only mutations into account, Weiss, et al. estimated a population frequency of compound heterozygosity of $0.34 \%$, which would represent an expected frequency of 3 compound heterozygotes out of our 949 cases, compared to the 8 compoud heterozygotes we did identify. As noted above, the functional importance of these additional variants varies in terms of their contribution to pulmonary disease, and their effect on pancreatic disease is essentially unknown. However, since none of the carriers of two mutations in our study had a clinical diagnosis of CF, it is likely that at least some of these mutations are not highly functional, at least with regard to pulmonary disease. Therefore, since the functional importance of each of the mutations in the 39-site panel is well established in the CF literature, we analyzed all 50 carriers as one group of "carriers" of at least 1 risk allele, whether or not they carried a second mutation of unclear functionality.

Of interest, none of our detected compound heterozygotes reported CF in their medical records, none had known long-standing pancreatitis, and they were older at diagnosis than other carriers. The importance of carrying a second mutation will require further study.

\section{Limitations}

This study has several limitations. First, the control group and patient group differed substantially in age and sex distribution. The absence of smoking data on controls also did not allow us to adjust for this known risk factor. However, we believe that the large sample size of the control group provides a reasonable population sample. The carrier rate is similar to that expected for persons of European ancestry (13,37-39). Another limitation is that CFTR assays have been developed for prenatal/prepregnancy screening purposes to detect parents at risk for having children with pulmonary disease. It is possible that the pancreatic disease in CF patients occurs through impairment of a different function of the CFTR gene than pulmonary disease. For instance, mutations that affect the bicarbonate exchange function of CFTR appear to be those more associated with pancreatic insufficiency (40). Full gene sequencing of all cases and controls may be required to begin to address this question in future studies. This study was not intended to replicate our findings from our pilot study of young onset cases. In this expanded study, we intended to explore the overall effect in our patient population including older onset patients and a larger sample to more fully define the role of CFTR mutations in pancreatic cancer.

With any case-control study, biases are possible. However, we believe the likelihood of increased referral or self-referral on the basis of genotype (and possible family history of CF) is low. Most carriers of mutations are likely unaware. On a self-completed clinical intake form, patients were asked if they have a family history of a "genetic disorder." Among the 50 mutation carriers, 48 completed the form. All 39 who answered the question reported "None."

\section{Conclusion}

Carrying a germline mutation in CFTR modestly increases risk for pancreatic adenocarcinoma. Mutation carriers also appear to be diagnosed at a younger age than noncarriers, with this effect seen exclusively among ever-smokers. Long-standing pancreatitis is uncommon regardless of carrier status.

\section{Abbreviations}

CF $\quad$ cystic fibrosis 
CFTR cystic fibrosis transmembrane conductance regulator [gene]

CI confidence interval

OR odds ratio

\section{Acknowledgments}

Sources of funding for this research: Lustgarten Foundation for Pancreatic Cancer Research, National Cancer Institute grants R01 CA97075 and CA116303, and Mayo Clinic SPORE in Pancreatic Cancer (P50 CA 102701). The authors acknowledge no conflicts of interest.

\section{References}

1. Riela A, Zinsmeister AR, Melton LJ 3rd, et al. Increasing incidence of pancreatic cancer among women in Olmsted County, Minnesota, 1940 through 1988. Mayo Clin Proc 1992;67:839-845. [PubMed: 1434927]

2. Jemal A, Tiwari RC, Murray T, et al. Cancer statistics, 2004. CA Cancer J Clin 2004;54:8-29. [PubMed: 14974761]

3. Petersen GM, Hruban RH. Familial pancreatic cancer: where are we in 2003? J Natl Cancer Inst 2003;95:180-181. [PubMed: 12569133]

4. Sharer N, Schwarz M, Malone G, et al. Mutations of the cystic fibrosis gene in patients with chronic pancreatitis. N Engl J Med 1998;339:645-652. [PubMed: 9725921]

5. Durno C, Corey M, Zielenski J, et al. Genotype and phenotype correlations in patients with cystic fibrosis and pancreatitis. Gastroenterology 2002;123:1857-1864. [PubMed: 12454843]

6. Lowenfels AB, Maisonneuve P, Cavallini G, et al. International Pancreatitis Study Group. Pancreatitis and the risk of pancreatic cancer. N Engl J Med 1993;328:1433-1437. [PubMed: 8479461]

7. Tsongalis GJ, Faber G, Dalldorf FG, et al. Association of pancreatic adenocarcinoma, mild lung disease, and $\Delta$ F508 mutation in a cystic fibrosis patient. Clin Chem 1994;40:1972-1974. [PubMed: 7522998]

8. Davis TM, Sawicka EH. Adenocarcinoma in cystic fibrosis. Thorax 1985;40:199-200. [PubMed: 3983888]

9. McIntosh JC, Schoumacher RA, Tiller RE. Pancreatic adenocarcinoma in a patient with cystic fibrosis. Am J Med 1988;85:592. [PubMed: 3177424]

10. SEER*Stat Database: Incidence - Seer 9 Regs Public-Use, Nov 2002 Sub (1973-2001) [Internet]. Surveillance, Epidemiology, and End Results (SEER) Program. 2003- [cited 2004 Jan]. Available from: http://www.seer.cancer.gov.

11. Sheldon CD, Hodson ME, Carpenter LM, et al. A cohort study of cystic fibrosis and malignancy. $\mathrm{Br}$ J Cancer 1993;68:1025-1028. [PubMed: 8217592]

12. Neglia JP, FitzSimmons SC, Maisonneuve P, et al. Cystic Fibrosis and Cancer Study Group. The risk of cancer among patients with cystic fibrosis. N Engl J Med 1995;332:494-499. [PubMed: 7830730]

13. McWilliams RR, Highsmith WE, Rabe KG, et al. Cystic fibrosis transmembrane regulator gene carrier status is a risk factor for young onset pancreatic adenocarcinoma. Gut 2005;54:1661-1662. [PubMed: 16227367]

14. Wang L, Bamlet WR, de Andrade M, et al. Mitochondrial genetic polymorphisms and pancreatic cancer risk. Cancer Epidemiol Biomarkers Prev 2007;16:1455-1459. [PubMed: 17627010]

15. Amos JA, Bridge-Cook P, Ponek V, et al. A universal array-based multiplexed test for cystic fibrosis carrier screening. Expert Rev Mol Diagn 2006;6:15-22. [PubMed: 16359263]

16. Farrell PM, Rosenstein BJ, White TB, et al. Cystic Fibrosis Foundation. Guidelines for diagnosis of cystic fibrosis in newborns through older adults: Cystic Fibrosis Foundation consensus report. J Pediatr 2008;153:S4-S14. [PubMed: 18639722]

17. Strom CM, Janeszco R, Quan F, et al. Technical validation of a TM Biosciences Luminex-based multiplex assay for detecting the American College of Medical Genetics recommended cystic fibrosis mutation panel. J Mol Diagn 2006;8:371-375. [PubMed: 16825511] 
18. Johnson MA, Yoshitomi MJ, Richards CS. A comparative study of five technologically diverse CFTR testing platforms. J Mol Diagn 2007;9:401-407. [PubMed: 17591940]

19. Ahmed N, Corey M, Forstner G, et al. Molecular consequences of cystic fibrosis transmembrane regulator $(C F T R)$ gene mutations in the exocrine pancreas. Gut 2003;52:1159-1164. [PubMed: 12865275]

20. Shoshani T, Augarten A, Gazit E, et al. Association of a nonsense mutation (W1282X), the most common mutation in the Ashkenazi Jewish cystic fibrosis patients in Israel, with presentation of severe disease. Am J Hum Genet 1992;50:222-228. [PubMed: 1370365]

21. Malats N, Casals T, Porta M, et al. Cystic fibrosis transmembrane regulator (CFTR) $\triangle \mathrm{F} 508$ mutation and $5 \mathrm{~T}$ allele in patients with chronic pancreatitis and exocrine pancreatic cancer. Gut 2001;48:7074. [PubMed: 11115825]

22. Matsubayashi H, Fukushima N, Sato N, et al. Polymorphisms of SPINK1 N34S and CFTR in patients with sporadic and familial pancreatic cancer. Cancer Biol Ther 2003;2:652-655. [PubMed: 14688470]

23. Bobadilla JL, Macek M Jr, Fine JP, et al. Cystic fibrosis: a worldwide analysis of CFTR mutations: correlation with incidence data and application to screening. Hum Mutat 2002;19:575-606. [PubMed: 12007216]

24. Layer P, DiMagno EP. Early and late onset in idiopathic and alcoholic chronic pancreatitis: different clinical courses. Surg Clin North Am 1999;79:847-860. [PubMed: 10470331]

25. Lowenfels AB, Maisonneuve P, Whitcomb DC, et al. Risk factors for cancer in hereditary pancreatitis. Med Clin North Am 2000;84:565-573. [PubMed: 10872414]

26. Ferec C, Novelli G, Verlingue C, et al. Identification of six novel CFTR mutations in a sample of Italian cystic fibrosis patients. Mol Cell Probes 1995;9:135-137. [PubMed: 7541510]

27. Cuppens H, Jaspers M, Lin W, et al. Exon 10 skipping resulting from the E528E polymorphism [abstract]. Pediatric Pulmonology 1998;26:302.

28. Clain J, Lehmann-Che J, Girodon E, et al. A neutral variant involved in a complex CFTR allele contributes to a severe cystic fibrosis phenotype. Hum Genet 2005;116:454-460. [PubMed: 15744523]

29. Padoan R, Genoni S, Moretti E, et al. Genetic and clinical features of false-negative infants in a neonatal screening programme for cystic fibrosis. Acta Paediatr 2002;91:82-87. [PubMed: 11883825]

30. Boyne J, Evans S, Pollitt RJ, et al. Many $\Delta$ F508 heterozygote neonates with transient hypertrypsinaemia have a second, mild CFTR mutation. J Med Genet 2000;37:543-547. [PubMed: 10970190]

31. Monaghan KG, Highsmith WE, Amos J, et al. Genotype-phenotype correlation and frequency of the 3199del6 cystic fibrosis mutation among I148T carriers: results from a collaborative study. Genet Med 2004;6:421-425. [PubMed: 15371907]

32. Wei L, Vankeerberghen A, Jaspers M, et al. Suppressive interactions between mutations located in the two nucleotide binding domains of CFTR. FEBS Lett 2000;473:149-153. [PubMed: 10812063]

33. Chu CS, Trapnell BC, Curristin S, et al. Genetic basis of variable exon 9 skipping in cystic fibrosis transmembrane conductance regulator mRNA. Nat Genet 1993;3:151-156. [PubMed: 7684646]

34. Chillon M, Casals T, Mercier B, et al. Mutations in the cystic fibrosis gene in patients with congenital absence of the vas deferens. N Engl J Med 1995;332:1475-1480. [PubMed: 7739684]

35. Bishop MD, Freedman SD, Zielenski J, et al. The cystic fibrosis transmembrane conductance regulator gene and ion channel function in patients with idiopathic pancreatitis. Hum Genet 2005;118:372381. [PubMed: 16193325]

36. Weiss FU, Simon P, Bogdanova N, et al. Complete cystic fibrosis transmembrane conductance regulator gene sequencing in patients with idiopathic chronic pancreatitis and controls. Gut 2005;54 (10):1456-1460. [PubMed: 15987793]

37. Gasparini P, Arbustini E, Restagno G, et al. Analysis of 31 CFTR mutations by polymerase chain reaction/oligonucleotide ligation assay in a pilot screening of 4476 newborns for cystic fibrosis. J Med Screen 1999;6:67-69. [PubMed: 10444722] 
38. Konialis CP, Hagnefelt B, Kazamia C, et al. CFTR $\triangle F 508$ mutation detection from dried blood samples in the first trimester of pregnancy: a possible routine prenatal screening strategy for cystic fibrosis? Fetal Diagn Ther 2007;22:41-44. [PubMed: 17003555]

39. Fries MH, Bashford M, Nunes M. Implementing prenatal screening for cystic fibrosis in routine obstetric practice. Am J Obstet Gynecol 2005;192:527-534. [PubMed: 15695998]

40. Choi JY, Muallem D, Kiselyov K, et al. Aberrant CFTR-dependent $\mathrm{HCO}_{3}^{-}$transport in mutations associated with cystic fibrosis. Nature 2001;410:94-97. [PubMed: 11242048] 


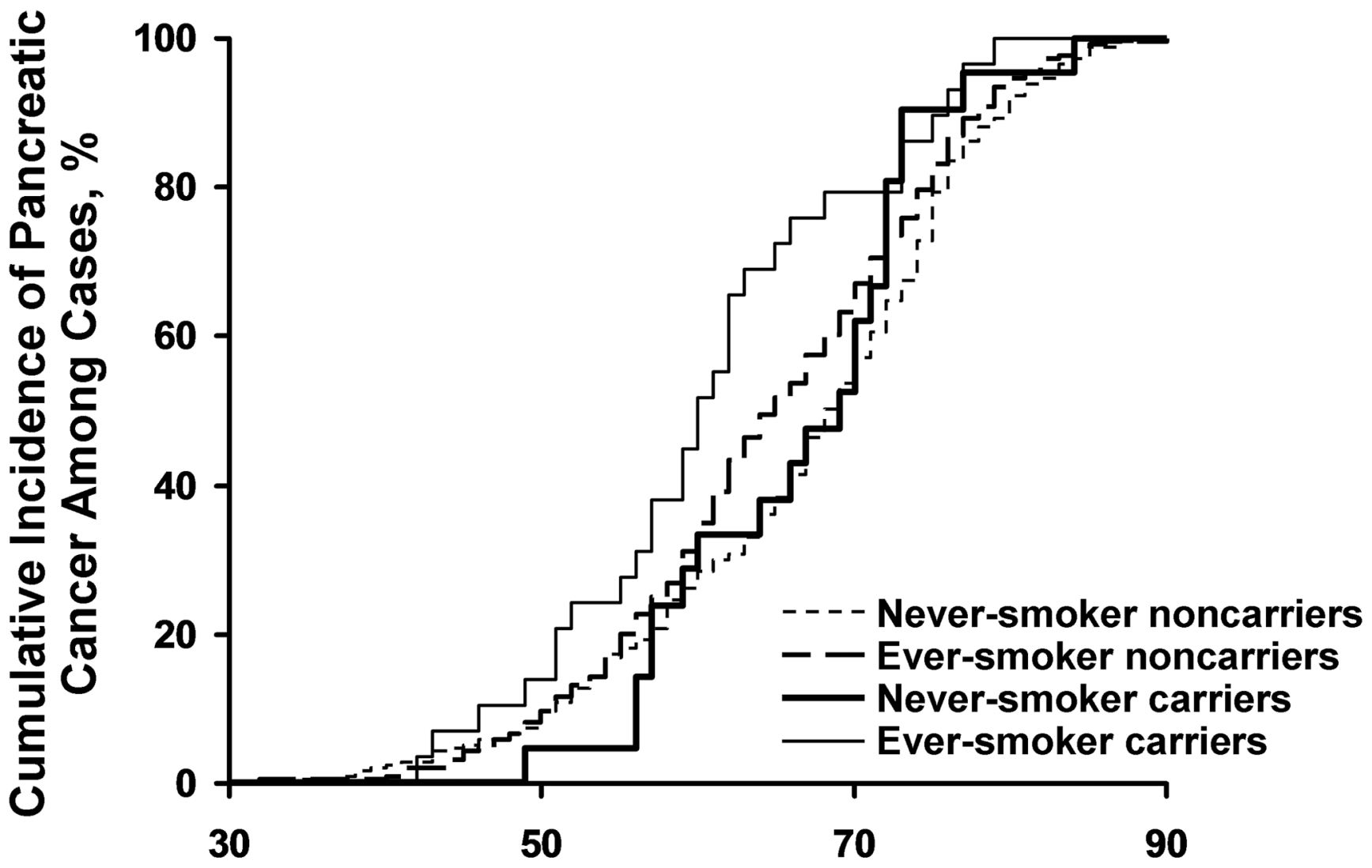

\section{Age at Diagnosis, y}

Figure 1.

Kaplan-Meier Estimates of Age at Diagnosis of Pancreatic Cancer. Kaplan-Meier curve of age at diagnosis of pancreatic cancer among ever-smokers and never-smokers by CFTR carrier status compares those with detected mutations with those without. The median age at pancreatic cancer diagnosis for carriers was 60 years for ever-smoker carriers, 65 years for ever-smoker noncarriers, 68 years for never-smoker noncarriers, and 69 years for never-smoker carriers $(P=.0019$ by log-rank analysis). 
Table 1

Comparison of CFTR Mutation Frequencies in Pancreatic Cancer Patients and Controls ${ }^{a}$

\begin{tabular}{|c|c|c|c|}
\hline CFTR Mutation & $\begin{array}{c}\text { No. (\%) of Patients } \\
(n=949)\end{array}$ & No. $(\%)$ of Controls $(n=13,340)$ & Significance \\
\hline Not detected & $899(94.7)$ & $12,830(96.2)$ & $\begin{array}{l}\text { OR, } 1.40(95 \% \\
\text { CI, } 1.04-1.89)\end{array}$ \\
\hline Detected & $50(5.3)$ & $510(3.8)$ & $P=.027$ \\
\hline$\Delta \mathbf{F 5 0 8} b$ & $35(70)$ & $354(69.4)$ & \\
\hline $\mathbf{R} 117 H^{c}$ & $5(10)$ & $71(13.9)$ & \\
\hline $\mathrm{G551D}^{b}$ & $1(2)$ & $11(2.2)$ & \\
\hline $\mathrm{N}^{1303 K^{b}}$ & $1(2)$ & $8(1.6)$ & \\
\hline $\mathrm{G542X}^{b}$ & & $8(1.6)$ & \\
\hline $1717-1 G>A^{b}$ & & $7(1.4)$ & \\
\hline $2789+5 G>A$ & & $6(1.2)$ & \\
\hline $\mathrm{R}^{253} \mathrm{X}^{b}$ & & $6(1.2)$ & \\
\hline $\mathrm{W} 1282 \mathrm{X}^{b}$ & $5(10)$ & $5(1.0)$ & \\
\hline $\mathrm{R} 347 \mathrm{H}$ & & $5(1.0)$ & \\
\hline $\mathrm{R}_{1162 X^{b}}$ & & $4(0.8)$ & \\
\hline $621+1 G>T^{b}$ & & $4(0.8)$ & \\
\hline $\mathrm{R}^{260 \mathrm{~T}^{b}}$ & & $3(0.6)$ & \\
\hline R347P & $1(2)$ & $2(0.4)$ & \\
\hline A455E & & $2(0.4)$ & \\
\hline $3849+10 \mathrm{kbC}>\mathrm{T}$ & & $2(0.4)$ & \\
\hline 394delTT & & $2(0.4)$ & \\
\hline G85E & & $2(0.4)$ & \\
\hline 3905 insT $^{b}$ & & $2(0.4)$ & \\
\hline $1898+1 G>A$ & & $2(0.4)$ & \\
\hline $2183 \mathrm{AA}>\mathrm{G}$ & & $1(0.2)$ & \\
\hline 2184delA ${ }^{b}$ & & $1(0.2)$ & \\
\hline $711+1 G>T^{b}$ & & $1(0.2)$ & \\
\hline $\mathrm{V} 520 \mathrm{~F}$ & & $1(0.2)$ & \\
\hline $\mathrm{S} 549 \mathrm{R}^{b}$ & $1(2)$ & & \\
\hline$\Delta \mathrm{I507} b$ & $1(2)$ & & \\
\hline
\end{tabular}

Abbreviations: CI, confidence interval; OR, odds ratio.

${ }^{a}$ Bold font indicates mutations recommended for screening by the American College of Medical Genetics (16). Mutations not listed but included in the 39-site assay: 3120 +1G>A, R334W, 3569delC, 1078delT, S549N, 3876delA, 1898+5G>T, 2307insA, Y1092X, M1101K, S1255X, Y122X, A559T; in the 33-site assay: 3120 +1G >A, R334W, 3569delC, S549N, 3876delA, F508C.

${ }^{b} \mathrm{CF}$ patients with these genotypes have more severe pancreatic insufficiency (19).

${ }^{c}$ All cases were homozygous for the $7 \mathrm{~T}$ allele of the poly-T polymorphism. 


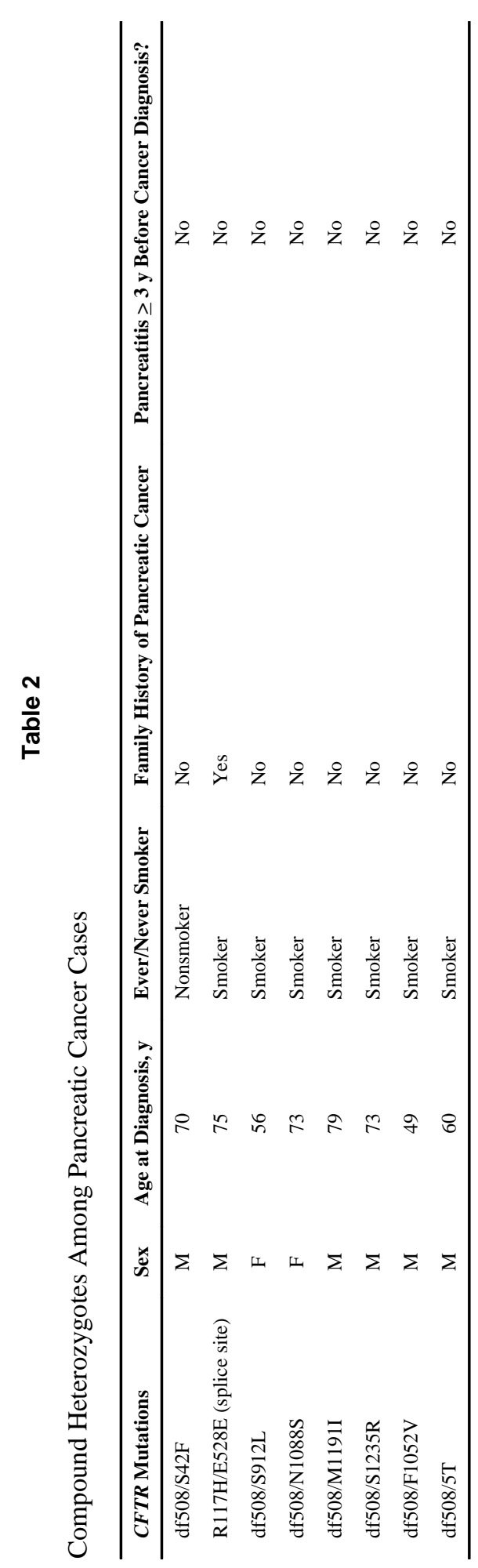

Cancer. Author manuscript; available in PMC 2011 January 1. 